\title{
DESENVOLVIMENTO DE UM MODELO PARA O BIOSPECKLE NA ANÁLISE DE SEMENTES DE FEIJÃO (Phaseolus vulgaris L.)
}

\author{
Development of a model for the biospeckle applied to analise been seed \\ (Phaseolus vulgaris L.)
}

\author{
Álvaro Leonardo do Nascimento 1 , Antonio Tavares Costa Júnior², \\ Giovanni Francisco Rabelo ${ }^{3}$, Roberto Alves Braga Júnior ${ }^{4}$
}

\begin{abstract}
RESUMO
O biospeckle é uma figura de interferência formada pela reflexão difusa da luz coerente espalhada ao interagir com um objeto que apresenta algum tipo de atividade, biológica ou não. O padrão de interferência se modifica ao longo do tempo devido às estruturas responsáveis pelo espalhamento estarem em atividade. Esse fenômeno tem sido estudado com o intuito de se desenvolver um método rápido e não destrutivo para avaliação de materiais biológicos. A obtenção de um modelo simples que descreva os aspectos essenciais do fenômeno é um importante passo para o domínio da técnica. Neste trabalho é apresentado um modelo para descrever a formação do biospeckle, desenvolvido a partir de hipóteses simples sobre como o tecido biológico difrata a luz coerente e qual o efeito da atividade biológica sobre a difração. Foram comparados os resultados da simulação do modelo com resultados experimentais obtidos de sementes. O modelo reproduz com sucesso algumas das características básicas do padrão dinâmico.
\end{abstract}

Termos para indexação: Speckle, difração, óptica de Fourier, atividade biológica.

\begin{abstract}
Biospeckle is an interference pattern phenomenon formed by the diffuse reflection of coherent light scattered by any type of activity, biological or not. The interference pattern changes in time due to the movement of the structures that scatter the light. This phenomenon has been studied with the goal of developing a fast and non-destructive method for evaluating biological material. Building a simple model that describes the essential aspects of the phenomenon is an important step towards the development of this technique. In this work is presented a model that describes the formation of the biospeckle, based on simple assumptions about the scattering of coherent light by the biological material and the nature of biological activity. We compare the results provided by the model with experimental biospeckle obtained from seeds. The model reproduces successfully some of the basic characteristics of the dynamical experimental pattern.
\end{abstract}

Index terms: Speckle, diffraction, Fourier optics, biological activity.

(Recebido em 2 de março de 2005 e aprovado em 9 de setembro de 2005)

\section{INTRODUÇÃO}

Com a necessidade de técnicas rápidas e eficientes de avaliação de propriedades de materiais biológicos, vêm sendo destacadas as que utilizam métodos não destrutivos, uma vez que preservam o produto analisado. Os testes atuais para análise de vigor e viabilidade de sementes têm um desafio que é a capacidade, de em apenas um teste, indicar o desempenho da semente baseando-se nas distintas características avaliadas pelos mesmos (MARCOS FILHO, 1999).

Os métodos que empregam visão artificial e processamento de imagens ocupam um lugar de destaque em pesquisas (HOWARTH \& STANWOOD, 1993). Nesse sentido, trabalhos como o de Neurohr et al. (1991) buscaram correlacionar a emissão de luz em sementes, por meio da emissão fluorescente ("delayed light emission"), com aspectos de qualidade. Uma técnica que vem surgindo como forma de avaliação de atividade biológica baseia-se em um fenômeno óptico de interferência que ocorre quando a luz laser incide sobre uma superfície onde se desenvolve um processo dinâmico. Esse fenômeno é conhecido como speckle dinâmico ou biospeckle. A técnica do biospeckle foi apresentada como uma metodologia potencial para a análise de sementes, porém indicando a necessidade de maior domínio dos diferentes fatores que podem estar interferindo no fenômeno (RABAL et al., 1998).

\footnotetext{
'Engenheiro Agrícola, Mestre em Máquinas e Automação Agrícola, Universidade Federal de Lavras/UFLA - Cx..P. 3037 - $37200-000$ - Lavras, MG leo@stout.ufla.br

${ }^{2}$ Físico, Professor do Departamento de Ciências Exatas da Universidade Federal de Lavras/UFLA - Cx..P. 3037 - $37200-000$ - Lavras, MG antc@stout.ufla.br

${ }^{3}$ Engenheiro Eletricista, Professor do Departamento de Engenharia da Universidade Federal de Lavras/UFLA - Cx..P. 3037 - $37200-000$ - Lavras, MG giovannirabelo@yahoo.com.br

${ }^{4}$ Engenheiro Eletricista, Professor do Departamento de Engenharia da Universidade Federal de Lavras/UFLA - Cx..P. 3037 - $37200-000$ - Lavras, MG roberto@ufla.br
} 
Ao incidir e penetrar em um material biológico, a luz laser sofre espalhamentos múltiplos pelas estruturas microscópicas presentes na superfície e em camadas subsuperficiais do tecido (RABELO, 2000). O padrão formado pela luz espalhada é uma figura de interferência complexa que se modifica com o tempo, pois as estruturas responsáveis pelo espalhamento estão em constante atividade, que lhes modifica as propriedades ópticas. Desde a sua descoberta, esforços têm sido empenhados, na exploração do biospeckle como ferramenta não destrutiva para avaliar a atividade biológica.

Estudos das propriedades estatísticas da história temporal da intensidade do speckle, utilizando a função de autocorrelação do Padrão Temporal e Espacial do Speckle (Spatial Temporal Speckle-STS) como ferramenta, foram apresentados por Rabelo (2000) e Romero (1999). Essa ferramenta já havia sido utilizada também por Bergkvist (1997), Oulamara (1989) e Xu et al. (1995).

Outra análise das propriedades do STS é o cálculo do módulo de dispersão de intensidades, também denominado, por alguns autores como momento de inércia (mi), que é obtido por meio da Matriz de Ocorrência (MOC), proposto por Arizaga et al. (1999).

Devido à complexidade do biospeckle seria muito conveniente dispor de um modelo que descrevesse ao menos as características principais do fenômeno (BRAGA JÚNIOR, 2000).

Objetivou-se com este trabalho elaborar o modelo que reproduza o speckle dinâmico proveniente da exposição de sementes à luz laser. Neste trabalho, utilizouse sementes de feijão (Phaseolus vulgaris L.).

\section{MATERIAIS E MÉTODOS}

Do ponto de vista do espalhamento de luz, o tecido biológico pode ser visto como um conjunto de estruturas ou organelas, que refletem a luz incidente separadas por espaços vazios, que absorvem a luz incidente. $\mathrm{O}$ processo de espalhamento da luz por cada organela do tecido é bastante complexo. Entretanto, para a elaboração do modelo, despreza-se esta complexidade e supõe-se que cada organela reflete a luz incidente, como se fosse um objeto refletor plano, e os espaços vazios absorvem completamente a luz incidente. Como a atividade do biospeckle é característica mais relevante para a maioria das aplicações, este será o aspecto que nosso modelo tentará reproduzir. Assim, as "organelas" do modelo serão objetos planos que executam um movimento aleatório, resultado de sua atividade biológica. Este não é, entretanto, um movimento aleatório simples. As organelas da superfície do tecido deslocam-se em torno de uma região restrita, delimitada pela presença das outras organelas. O sistema de organelas executa um passeio aleatório no qual as colisões entre elas são levadas em conta. Isto faz com que as trajetórias das várias organelas estejam correlacionadas.

Em resumo, no modelo proposto para o tecido biológico este é composto por um conjunto de objetos planos executando passeios aleatórios com barreiras refletoras, que refletem toda a luz que incide sobre eles, sem alteração de fase.

O padrão resultante do espalhamento de luz por esta estrutura de fendas, observado a uma distância relativamente grande comparada com o tamanho das fendas corresponde ao padrão de difração de Fraunhoffer de um conjunto de fendas retangulares, que pode ser calculado através da transformada de Fourier da função de reflectância do padrão (HECHT, 2002). Esta, por ser dinâmica, resultará num padrão dependente do tempo,

$$
I(x, t)=\frac{1}{2 \pi} \int_{-\infty}^{\infty} d x e^{-i k x} R(x, t)
$$

em que $t$ é o tempo, $x$ é a posição sobre o padrão de speckle, $k$ a posição no tecido e $R(x, \mathrm{t})$ é a função de reflectância. $\mathrm{O}$ modelo $R(x, t)$, proposto neste trabalho, é representado pela equação 2 .

$$
R(x, t)=\sum_{i=1}^{N_{f}} \prod_{b_{i}}\left[x-a_{i}(t)\right]
$$

em que

$$
\prod_{b}(x)= \begin{cases}1, & \text { se }-b<x<b \\ 0, & \text { caso contrário }\end{cases}
$$

$N_{f}$ é o número de fendas, $b_{i}$, e $a_{i}(t)$ são a largura e a posição da fenda $i$, respectivamente. A posição da fenda $i$ segue um passeio aleatório dado por:

$$
\mathrm{a}_{\mathrm{i}}(\mathrm{t}+\Delta \mathrm{t})=\mathrm{a}_{\mathrm{i}}(\mathrm{t})+\mathrm{S}_{\mathrm{i}}
$$

em que $S_{i}$, é o tamanho do passo, restrito a um intervalo que é delimitado pela presença de outra fenda. Note que o tamanho de cada passo é um parâmetro ajustável no modelo, e corresponde a uma medida global de atividade biológica do tecido. Um tecido que apresenta uma atividade menos intensa deve ter organelas que se movem com menor velocidade, e vice-versa.

A princípio é possível obter resultados experimentais para este valor, mas isso envolveria um estudo dinâmico de microscopia eletrônica de vários tecidos biológicos diferentes, o que está fora das capacidades 
experimentais disponíveis no momento. De qualquer modo, este é o único parâmetro ajustável no modelo que tem influência direta sobre a atividade biológica.

Os valores das larguras das fendas e o espaçamento máximo entre elas foram escolhidos com base em dados experimentais de microscopia sobre o tamanho médio das partículas e seu espaçamento médio em sementes. O tamanho típico das partículas é da ordem de $10^{-5} \mathrm{~m}$, e o espaçamento $10^{-6} \mathrm{~m}$.

Do padrão resultante escolhe-se um ponto (pixel), do qual se tomam intensidades em cada instante. Isto corresponde a analisar a intensidade de um ponto do biospeckle. A análise da atividade simulada pelo modelo pode ser de duas maneiras. Uma baseia-se na interpretação da função de autocorrelação temporal de um ponto arbitrário do biospeckle, a outra é pelo valor do momento de inércia (mi).

O módulo de dispersão de intensidades é dado pelo somatório das ocorrências de uma intensidade $i$ seguida por uma intensidade $j$, multiplicada pelo quadrado da diferença de suas posições, segundo equação 4 .

$$
M I=\sum_{i j} M_{i j}(i-j)^{2}
$$

em que $M_{\mathrm{ij}}$ é a matriz de ocorrência de intensidades de níveis de cinza representados por $i$ e $j$.

A função autocorrelação temporal da intensidade de um elemento da imagem (pixel), é a medida do valor médio do produto de duas intensidades separadas por um instante de tempo $t$ (equação 5).

$$
\rho(\tau)=\frac{1}{N} \sum_{i=1}^{N} I\left(t_{i}+\tau\right) I\left(t_{i}\right)
$$

em que I(t) representa o vetor de intensidades do sinal que será convoluído, e que fornece a autocorrelação, com o $\tau$ sendo o passo de convolução e $\mathrm{N}$ o número de pontos do sinal.

A autocorrelação fornece uma medida indireta da atividade biológica do modelo. Um tecido estático produziria um espectro sem variação temporal, e a correlação temporal seria máxima e constante para quaisquer variações rápidas de intensidade, e, como se supõe os movimentos dos vários componentes do tecido descorrelacionados, a autocorrelação temporal deve cair rapidamente com o intervalo $\tau$.

Para obter resultados a partir do modelo foi criado um programa computacional, que simula o passeio aleatório com barreiras refletoras de várias fendas, e calcula o padrão de difração de Fraunhoffer do sistema a cada passo temporal em um ponto fixo arbitrário, a uma distância de um metro das fendas. De posse das intensidades deste ponto do padrão para cada instante de tempo, calcula-se a função de autocorrelação temporal do padrão. $\mathrm{O}$ ajuste do parâmetro livre do modelo, o tamanho do passo das fendas, é feito por comparação entre autocorrelações experimentais e as obtidas a partir do modelo. Como o objetivo é comparar os resultados do modelo com os resultados experimentais apenas qualitativamente, não é necessário utilizar métodos sofisticados de ajuste.

Esses resultados foram comparados com as funções de autocorrelação calculadas para biospeckle reais, obtidos experimentalmente a partir da iluminação com laser de sementes de feijão (Phaseoulus vulgaris L.) em diferentes níveis de umidade (13, 20 e $46 \%$ b.u.). Para cada umidade foram iluminadas 15 sementes.

Na Figura 1 pode-se observar a montagem utilizada para a iluminação e aquisição das imagens.

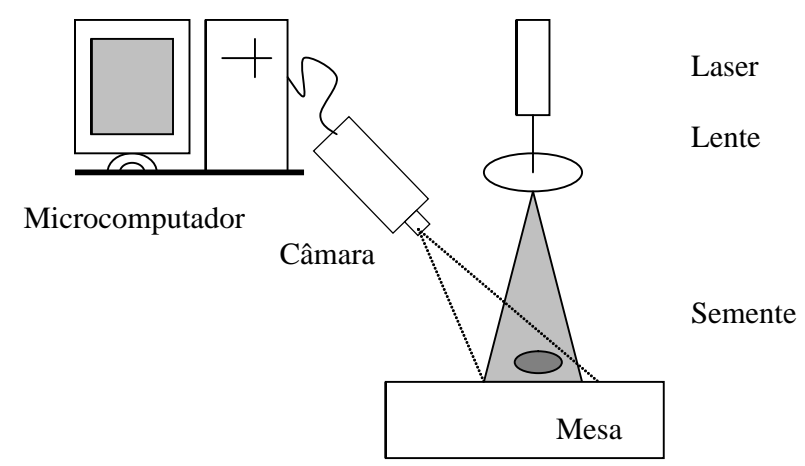

FIGURA 1 - Diagrama esquemático dos sistemas de iluminação laser e aquisição de imagens.

O laser de $\mathrm{He}-\mathrm{Ne}$, de $632 \mathrm{~nm}$, passando por um expansor de feixe ilumina o lado interno de um cotilédone da semente, e o speckle formado é capturado a uma taxa de 12,5 imagens/s por uma câmara digital. A autocorrelação é calculada da matriz conhecida por padrão espacial e temporal do speckle (STS). Para formação do STS é necessário obter imagens sucessivas do speckle no tempo. De posse dessas imagens retira-se uma mesma coluna das imagens capturadas, e com as colunas das imagens formase uma nova imagem NxM. Esta nova imagem, que representa as $\mathrm{N}$ colunas ao longo do tempo denomina-se de STS, que normalmente tem uma composição $\mathrm{N}=\mathrm{M}$. Neste caso as linhas representam a variação temporal de um pixel, e as colunas, a variação espacial no mesmo instante. Para o cálculo do momento de inércia faz-se o tratamento do STS o que dá origem à outra imagem denominada matriz de ocorrências (MOC). A MOC é uma matriz 256 x 256 que representa o número de situações em que ocorreu a 
mudança de um tom de cinza $i$ para um tom de cinza $j$, que pode variar de 0 a 255 tons de cinza. Sendo assim um algoritmo conta no STS o número de ocorrências que um pixel da imagem com determinado valor de intensidade $i$ é seguido por outro pixel que apresenta um outro valor de intensidade $j$. Este método foi proposto por Arizaga et al. (1999) e utilizado por outros autores como Braga Júnior (2000) e Rabelo (2000). A equação 6 apresenta a fórmula do cálculo da MOC.

$$
\mathrm{MOC}=\mathrm{N}_{\mathrm{ij}}
$$

em que $N$ representa o número de ocorrências de sucessivos pixels com intensidade $i$ seguida pela intensidade $j$.

Em uma matriz que o material apresenta baixa atividade, as ocorrências encontram-se próximo da diagonal principal, Figura 2(a), já para um material de alta atividade as ocorrências ficam dispersas pela matriz, Figura 2(b).

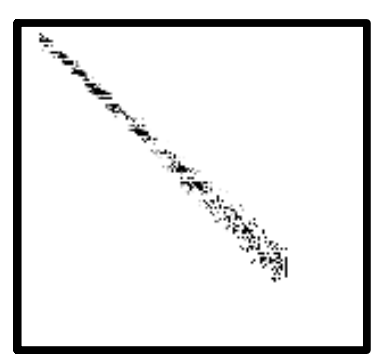

$2 \mathrm{a}$

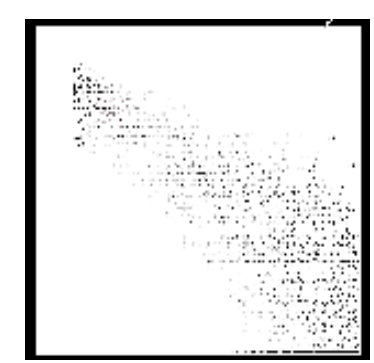

$2 b$
FIGURA 2 - Matriz de ocorrências de baixa atividade (2a) e alta atividade (2b) geradas a partir de biospeckles experimentais.

\section{RESULTADOS E DISCUSSÃO}

O modelo proposto demonstra uma grande capacidade de reproduzir diferentes resultados de funções de autocorrelação, desde funções em que os coeficientes caem lentamente até aquelas funções onde os coeficientes caem rapidamente, como apresentado na Figura 3.

Inspecionando-se as funções de autocorrelação geradas pelo modelo nota-se que este modelo é capaz de simular diferentes níveis de atividade. O mesmo é observado pelas matrizes de ocorrências geradas das simulações.

Na Figura 5, mostra-se a variação do momento de inércia do padrão de acordo com a variação do tamanho do passo. É possível perceber que há uma tendência de aumento do momento de inércia com o aumento do passo, o que concorda com a interpretação microscópica da atividade biológica.

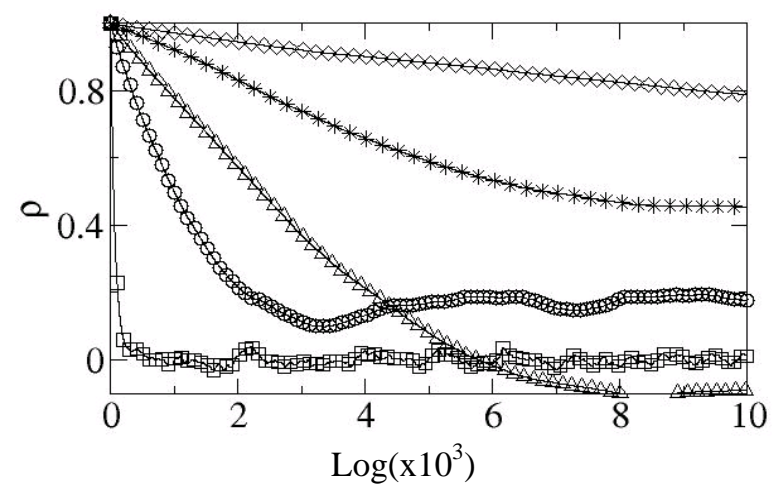

FIGURA 3 - Funções de autocorrelação $(\rho)$ ao longo do tempo provenientes do modelo, geradas pela variação do passo de $1 \times 10^{-8}$ até $5 \times 10^{-7}$.

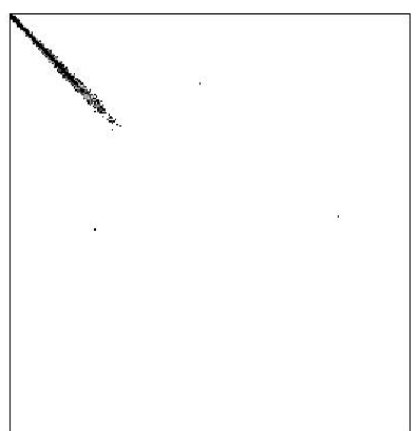

(a)

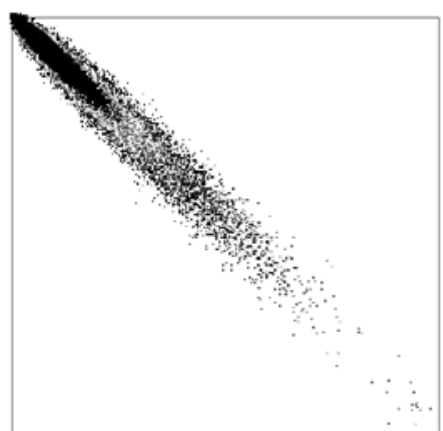

(b)

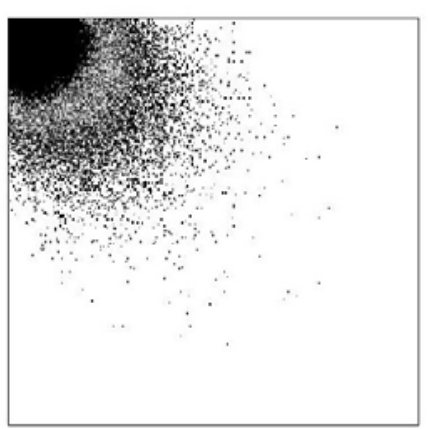

(c)

FIGURA 4 - Matrizes de ocorrências geradas pelo modelo representando: (a) baixa atividade, (b) média atividade, (c) alta atividade. 


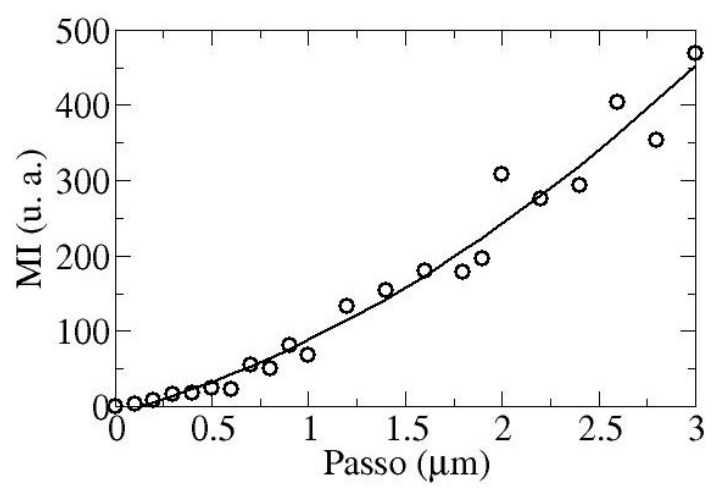

FIGURA 5-Tendência dos valores de momento de inércia, em unidade adimensionais,gerados pelo modelo, como função do passo $(\mu \mathrm{m})$.

No gráfico da Figura 6 são apresentados os ajustes das funções de autocorrelação geradas pelo modelo e as curvas provenientes das iluminações de feijão nos teores de umidade 13, 20 e $46 \%$ b.u.. É possível observar a concordância das curvas do modelo com as curvas do experimento. Os passos que melhor ajustaram os resultados do modelo aos experimentais foram o de $5 \times 10^{-7} \mathrm{~m}, 7,5 \times 10^{-7} \mathrm{~m}$ e $10 \times 10^{-7} \mathrm{~m}$ para os três níveis de umidade estudados, respectivamente. Estes valores são da mesma ordem de grandeza dos tamanhos das partículas e do espaçamento médio entre elas; isto confere coerência entre as hipóteses do modelo e os resultados obtidos.

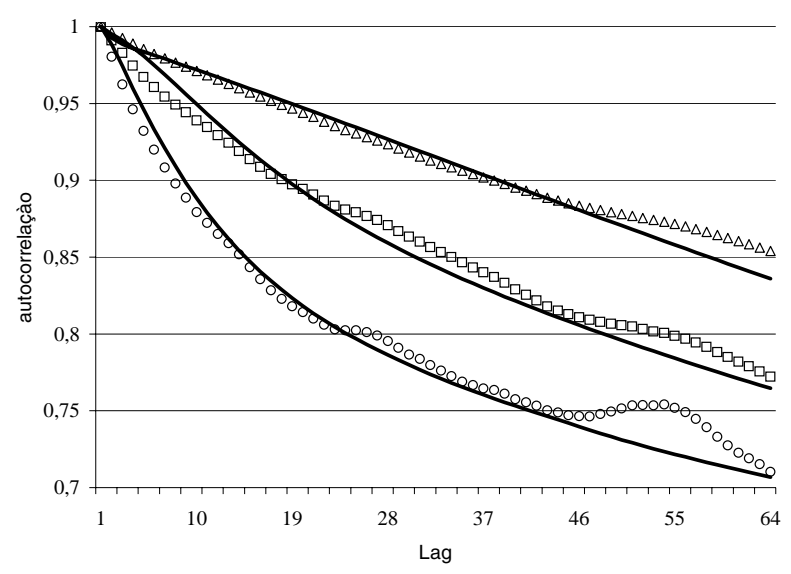

FIGURA 6 - Funções de autocorrelação $(\rho)$ das sementes iluminadas e das simulações referentes aos passos 1,0x10${ }^{6} \mathrm{~m}, 7,5 \times 10^{-7} \mathrm{~m}$ e $5,0 \times 10^{-7} \mathrm{~m}$, respectivos.
As comparações realizadas demonstram que o modelo proposto consegue reproduzir o aspecto básico do comportamento do tecido biológico, qual seja, sua atividade (correspondente, microscopicamente, ao movimento das partículas que compõem o tecido).

\section{CONCLUSÃO}

Este trabalho apresentou um modelo para a formação do biospeckle, baseado em hipóteses sobre o espalhamento de luz pelo tecido biológico e o comportamento microscópico dos centros dispersores no tecido. O modelo possui um parâmetro livre, que foi ajustado por comparação entre os resultados de simulações computacionais e resultados reais obtidos em sementes de três diferentes condições de umidade. O modelo, apesar de simples, consegue reproduzir qualitativamente bem as curvas de autocorrelação dos padrões de speckle experimentais. Trabalhos para incorporar outros aspectos do fenômeno, como a evaporação de água do tecido, encontram-se em andamento.

\section{REFERÊNCIAS BIBLIOGRÁFICAS}

ARIZAGA, R.; CAP, N.; RABAL, H. J. Speckle time evaluation characterization by coocorrence matrix analisis. Optics \& Laser Technology, Oxford, v. 31, n. 2, p. 163-169, Mar. 1999.

BERGKVIST, A. Biospeckle-based study of the line profile of light scattered in strawberries. 1997. $60 \mathrm{f}$. Dissertation (MSc) - Faculty of Tecnology at Lund University, Lund, 1997.

BRAGA JÚNIOR, R. A. Utilização do biospeckle laser em sementes: uma contribuição para o desenvolvimento de uma tecnologia. 2000. 117 f. Tese (Doutorado em Engenharia Agrícola) - Universidade Estadual de Campinas, Campinas, 2000.

HECHT, E. Óptica. Lisboa: Fundação Calouste Gulbenkian, 2002. $790 \mathrm{p}$.

HOWARTH, M. S.; STANWOOD, P. C. Tetrazolium staining viabiiity seed test using color image processing. Transactions of the ASAE, Saint Joseph, v. 36, n. 6, p. 1937-1940, Nov./Dec. 1993.

MARCOS FILHO, J. Testes de vigor: importância e utilização. In: KRZYZANOWSKI, F. C.; VIEIRA, R. D.; FRANÇA NETO, J. B. Vigor de sementes: conceitos e testes. Londrina: ABRATES, 1999. 
NEUROHR, R. et al. Photon emission: a new method for scanning the quality of food. Lebensmittel Rundschau, Deutsche, p. 78-82, 1991.

OULAMARA, A.; TRIBILLON, G.; DUVERNOY, J. Biological activity measurement on botanical specimen surfaces using a temporal decorrelation effect of laser speckle. Journal of Modern Optics, London, v. 36, n. 2, p. 165-179, Feb. 1989.

RABAL, H. J.; BRAGA JÚNIOR, R. A.; TRIVI, M. R.; DALFABBRO, I. M. O Uso do laser na agricultura. In: CONBEA. Energia, automação e instrumentação. Poços de Caldas, 1998.
RABELO, G. F. Avaliação do fenômeno "Biospeckle" no monitoramento de qualidade de laranjas (Citrus Sinensis). 2000. 149 f. Tese (Doutorado em Engenharia Agrícola) - Universidade Estadual de Campinas, Campinas, 2000.

ROMERO, G. G. Estudio y caracerization de patrones de speckle que varian en el tiempo. 1999. $133 \mathrm{f}$. Tese (Doutorado em Física) - Facultad de Ciencias Exactas, Universidade Nacional de Salta, Salta, 1999.

XU, Z.; JOENATHAN, C.; KHORANA, B. M. Temporal and spatial proprieties of the time-varing speckles of botanical specimens. Optical Engineering, Redondo Beach, v. 34, n. 5, p. 1487-1502, May 1995. 